\title{
Endometrial cavity fluid is associated with deleterious pregnancy outcomes in patients undergoing in vitro fertilization/ intracytoplasmic sperm injection: a retrospective cohort study
}

\author{
Wen-Xiu Zhang ${ }^{1,2,3,4,5 \#}$, Lian-Bao Cao ${ }^{2,3,4,5 \#}$, Ying $Z_{\text {hao }}^{2}$, Jing $\mathrm{Li}^{3}$, Bo-Feng $\mathrm{Li}^{3}$, Jia-Nan $\mathrm{Lv}^{3}$, Lei Yan ${ }^{2,3,4,5,6}$, \\ Jin-Long $\mathrm{Ma}^{2,3,4,5}$ \\ ${ }^{1}$ Qilu Hospitai (Qingdao), Cheeloo College of Medicine, Shandong University, Qingdao, China; ${ }^{2}$ School of Medcine, Cheeloo College of Medicine, \\ Shandong University, Jinan, China; ${ }^{3}$ Center for Reproductive Medicine, Cheeloo College of Medicine, Shandong University, Jinan, China; ${ }^{4}$ National \\ Research Center for Assisted Reproductive Technology and Reproductive Genetics, Jinan, China; ${ }^{5}$ The Key Laboratory of Reproductive Endocrinology \\ (Shandong University), Ministry of Education, Jinan, China; ${ }^{6}$ Shandong Provincial Key Laboratory of Reproductive Medicine, Jinan, China \\ Contributions: (I) Conception and design: JL Ma, L Yan; (II) Administrative support: JL Ma; (III) Provision of study materials or patients: J Li, BF Li, \\ JN Lv; (IV) Collection and assembly of data: WX Zhang, LB Cao, Y Zhao; (V) Data analysis and interpretation: Y Zhao, LB Cao; (VI) Manuscript \\ writing: All authors; (VII) Final approval of manuscript: All authors. \\ \#These authors contributed equally to this work. \\ Correspondence to: Lei Yan, MD. School of medicine, Shandong University, 44 Wenhuaxi Road, Jinan 250001, China. Email: yanlei@sdu.edu.cn.
}

Background: The effects of endometrial cavity fluid (ECF) on in vitro fertilization (IVF) and intracytoplasmic sperm injection (ICSI) pregnancy outcomes following embryo transfer (ET) are still controversial. We conducted the present study to investigate whether the presence of ECF in infertile patients scheduled to undergo IVF or ICSI was associated with pregnancy outcomes.

Methods: A retrospective cohort study design was used. Among infertile patients undergoing IVF/ICSI, those with and without ECF were matched 1:1 using propensity score matching (PSM). After ensuring that the baseline levels of the two matched groups were consistent, the pregnancy and obstetrical outcomes of the two groups were compared.

Results: Patients with ECF had significantly lower clinical rates of pregnancy $(1,061 / 1,862,57 \%$ vs. 1,182/1,862, 63.5\%; $\mathrm{P}<0.001)$, live birth $(902 / 1,862$, 48.4\% vs. 1,033/1,862, 55.5\%; $\mathrm{P}<0.001)$, biochemical pregnancy $(1,182 / 1,862,63.5 \%$ vs. $1,288 / 1,862,69.2 \% ; \mathrm{P}<0.001)$, and embryo implantation $(1,500 / 3,740$, $40.1 \%$ vs. 1,661/3,740, 44.4\%, $\mathrm{P}<0.001)$ than patients without ECF. Also, patients with ECF had a higher incidence of gestational diabetes $(17 / 78,22 \%$ vs. 8/94, 9\%, $\mathrm{P}=0.014)$. However, there were no differences in gestational weeks at delivery or birth weight between the two groups.

Conclusions: ECF was significantly associated with adverse pregnancy outcomes but showed no significant association with adverse obstetric outcomes (except for gestational diabetes).

Keywords: Endometrial cavity fluid (ECF); in vitro fertilization (IVF); intracytoplasmic sperm injection (ICSI); pregnancy outcomes; propensity score matching (PSM)

Submitted Apr 29, 2020. Accepted for publication Sep 29, 2020.

doi: 10.21037/atm-20-3623

View this article at: http://dx.doi.org/10.21037/atm-20-3623

\section{Introduction}

The interaction between the embryo and the intrauterine environment largely determines the success or failure of implantation, and successful implantation requires a receptive intrauterine environment for the embryos. There is a link between uterine pathology, decreased fertility, and spontaneous abortion (1). During in vitro fertilization (IVF) and embryo transfer (ET), the endometrial 
microenvironment is vital for successful implantation (2).

Endometrial cavity fluid (ECF) consists of blood, mucus, endometrial secretions, and/or tubal fluid (3). It has been hypothesized that embryonal apposition may not occur when a fluid layer covers the endometrium, and that the presence of ECF before ET in IVF cycles is associated with implantation failure $(4,5)$. Thus, excessive uterine fluid is detrimental to embryo implantation (4). One previous study found that if the fluid amount is $<3.5 \mathrm{~mm}$ in the anteriorposterior dimension, ECF will disappear by the day of ET, and, as a result, has no influence on embryo implantation. Furthermore, the clinical pregnancy rate is no worse than the patients without ECF (5). Another report concluded that if the ECF was not flushed backward from the hydrosalpinx during controlled ovarian hyperstimulation $(\mathrm{COH})$, there was no effect on the clinical pregnancy rate (6).

Collectively, the effects of the presence of ECF on IVF and intracytoplasmic sperm injection (ICSI) pregnancy outcomes following ET are still controversial. Here, we conducted a retrospective cohort study with a large sample size to investigate the relationship between ECF and IVF/ ICSI pregnancy outcomes and complications.

We present the following article in accordance with the strengthening the reporting of observational studies in epidemiology (STROBE) reporting checklist (available at http://dx.doi.org/10.21037/atm-20-3623).

\section{Methods}

\section{Ethical statement}

The study was approved by the Institutional Review Board of the Reproductive Hospital Affiliated to Shandong University \{[2018] IRB No. [47]\}. All procedures performed in this study involving human participants were in accordance with the Declaration of Helsinki (as revised in 2013). Due to the retrospective nature of the study informed consent was not required.

\section{Study design}

A total of 3,688 patients with ECF diagnosed by ultrasound who underwent IVF/ICSI and ET were screened from the electronic medical records of 21,548 patients who attended the Reproductive Hospital affiliated to Shandong University between January 2009 and December 2014, and whose follow-up was carried out by staff of the hospital between 2015 and 2016. ECF was detected and measured by ultrasound during $\mathrm{COH}$, and this continued until the final ultrasound examination before ET. A total of 1,034 patients were excluded based on the following criteria:

* ET was not carried out;

* Clinical information was incomplete;

* ECF was aspirated because of the presence of other uterine diseases or it absorbed spontaneously before ET;

* ECF $(>3.5 \mathrm{~mm}$ ) was aspirated before ET;

* Incomplete/loss of follow-up information.

Propensity score matching (PSM) was used as a method of group matching to ensure accurate matching and reduce the selection bias. In total, 2,654 patients with ECF were used to perform PSM matching. Patients in the matched group (according to PSM) met the following criteria:

* Underwent IVF/ICSI in ET cycles during the same period;

* ECF was not found during all pre-implantation ultrasonic examinations;

* Clinical information was complete;

* Follow-up information was complete.

Pregnancy outcomes in the two groups were blinded to the investigators during PSM.

The PSM (1:1) was performed according to the following variables:

* Age;

* Body mass index;

* Type of infertility;

* Indications for IVF/ICSI;

* Numbers of follicles in both ovaries;

* Baseline follicle-stimulating hormone (FSH), estrogen, and luteinizing hormone (LH) levels;

* Number of current cycle of IVF/ICSI;

* Protocol of $\mathrm{COH}$;

* Duration of gonadotropin-releasing hormone (GnRH) treatment;

* Starting dosage of GnRH;

* Total dosage of GnRH;

* Estradiol (E2) level on the day of triggering ovulation with human chorionic gonadotropin (hCG);

* Progesterone level on the hCG trigger day;

* Endometrial thickness on the hCG trigger day;

* Number of oocytes retrieved;

* Number of good-quality embryos (those with evensized blastomeres and $<10 \%$ fragments on day 2);

* Number of embryos transferred.

The type of infertility, indications for IVF/ICSI, and protocol of $\mathrm{COH}$ were treated as categorical variables (PSM 
tolerance $=0$ ), and the remaining variables were treated as quantitative variables (PSM tolerance $=0.01)$. After PSM, the successfully paired patients were regarded as an ECF group and a control group. The baseline levels of the two groups were compared before further analysis. Pregnancy outcomes, pregnancy complications, and obstetrical outcomes of the two groups were then analyzed.

\section{Luteal support, embryo culture, embryo evaluation, and ET}

All patients received a standardized ovarian stimulation protocol, oocyte retrieval, fertilization, and fresh ET of a day-3 embryo. Intramuscular progesterone at dose of $80 \mathrm{mg}$ /day was used for luteal support. Embryo culture, evaluation, and transfer were performed as previously described (7). Briefly, depending on the quality of the sperm, the oocytes were inseminated by conventional methods or ICSI approximately 4 to 6 hours after follicle aspiration. Morphological criteria were used for embryo scoring. On the third day, two high-quality embryos were selected for transfer.

The pregnancy outcome was defined as previously described, and included clinical pregnancy rate, biochemical pregnancy rate, live birth rate, and embryo implantation rate (7). Fetal indices including gestational age, birth weight, number of fetuses, and complications during pregnancy were classified as obstetrical outcomes.

\section{Statistical analysis}

Statistical analysis was performed using IBM SPSS Statistics (v. 24.0, IBM Corp., Armonk, NY, USA). The student's $t$-test, Chi-squared $\left(\chi^{2}\right)$ test, or Fisher's exact test were used to compare the outcomes of the two groups. Data are presented as the mean \pm standard deviation $(\mathrm{SD})$, and a $\mathrm{P}$ value $<0.05$ was considered statistically significant.

\section{Results}

The scheme of our study is shown in Figure 1. In all, 1,862 pairs of patients were matched successfully with PSM. Overall, the two groups had similar distributions of baseline variables (Table 1). The outcomes of $\mathrm{COH}$ in both groups are shown in Table 2. There were no significant differences in hormone levels (E2, progesterone), endometrial thickness on the day of triggering ovulation with hCG, dosage of $\mathrm{GnRH}$ used in $\mathrm{COH}$, and ET-related parameters between the two groups.
We studied the main outcomes of IVF/ICSI in the study and control groups. Overall, the biochemical pregnancy rate was significantly lower in the ECF group than in the control group $(1,182 / 1,862,63.5 \%$ vs. $1,288 / 1,862,69.2 \%$, respectively; $\mathrm{P}<0.001$, Table 3), and the relative risk (RR) of biochemical pregnancy of patients with ECF was 0.918 [95\% confidence interval (CI), 0.877-0.961], which had statistical significance. The clinical pregnancy rate in the ECF group was also lower than that in the control group $(1,061 / 1,862,57 \%$ vs. 1,182/1,862, $63.5 \%$, respectively; $\mathrm{P}<0.001$, Table 3); RR was 0.898 (95\% CI, 0.852-0.946). Moreover, the ECF group had increased incidences of adverse pregnancy outcomes (including abortion during a biochemical pregnancy and abortion during clinical pregnancy) compared with the control group, but the difference was not statistically significant (Table 3). The ECF group also had a lower live birth rate than the control group (902/1,862, 48.4\% vs. 1,033/1,862, 55.5\%, respectively; $\mathrm{P}<0.001$, Table 3); RR was 0.873 (95\% CI, 0.821-0.929). Furthermore, the embryo implantation rate in the ECF group was lower than that in the control group (1,500/3,740, 40.1\% vs. 1,661/3,740, 44.4\%, respectively; $\mathrm{P}<0.001$; Table 3); RR was 0.903 (95\% CI, 0.856-0.952).

We also found that the clinical pregnancy rate in patients with hydrosalpinx combined with ECF was significantly lower than that in patients with simple ECF (60/172, $34.8 \%$ vs. 842/1,690, 49.8\%, respectively; $\mathrm{P}=0.022$ ). In all patients with hydrosalpinx, a comparison between the ECF and control groups showed no significant difference in the clinical pregnancy rate $(63 / 1,862,3.4 \%$ vs. $50 / 1,862,2.7 \%$, respectively).

The obstetrical outcomes were also compared between the ECF and control groups to investigate whether the presence of ECF prior to implantation had any impact on fetal development and maternal health status during pregnancy. The fetal-related evaluation indices of both groups showed no significant differences. Pregnancyinduced hypertension had the highest incidence in both groups (Table 4). Also, gestational diabetes had a significantly higher incidence in the ECF group compared with the control group (17/78, $21.8 \%$ vs. 8/94, 9\%, respectively; $\mathrm{P}=0.014$; Table 4).

\section{Discussion}

Presently, the causes and effects of ECF remain unclear. Previous studies have found that the production of ECF may be related to pathological conditions such as hydrosalpinx, 


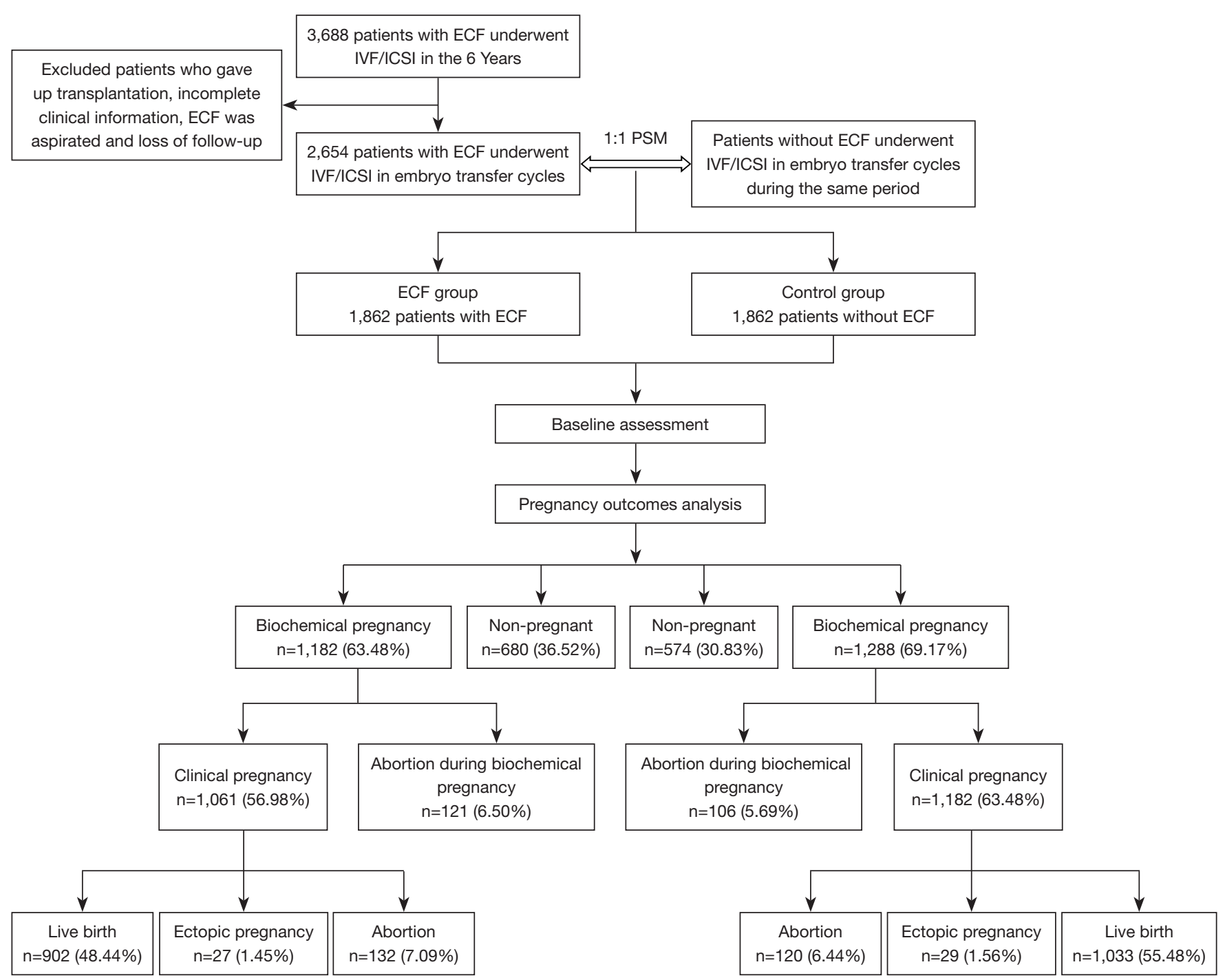

Figure 1 Experimental flow chart. The experimental process is described in detail in the study design. PSM, propensity score matching; ECF, endometrial cavity fluid; IVF, In vitro fertilization; ICSI, Intracytoplasmic sperm injection.

polycystic ovary disease, or subclinical uterine infection, or may be caused by the physiology of the reproductive tract (8). Following $\mathrm{COH}$, ovarian hyperstimulation syndrome (OHSS) is associated with extravasation of secretions and obstruction of the cervix leading to ECF, which can occur when there are cervical canal obstructions and pelvic adhesions (4).

Thus, the source and timing of ECF can be diverse. The primary focus of our study was to determine whether the presence of ECF is related to pregnancy outcomes. It has been shown that the presence of ECF during $\mathrm{COH}$ impairs the clinical pregnancy rate; however, the appearance of ECF after the hCG trigger does not (9). It is possible that ECF is closely related to hydrosalpinx, although the relationship between hydrosalpinx and pregnancy outcomes is controversial. Previous studies have reported that the flushing of hydrosalpinx fluids back into the endometrial cavity can interfere with embryo implantation $(10,11)$. Meanwhile, another study found that hydrosalpinx had no adverse effect on embryo development (12). There is an opinion that ECF, which is secreted by the genital tract and does not arise from the fallopian tube, has no effect on embryo implantation (6). Transient ECF is not associated with hydrosalpinx or any identifiable pelvic 
Table 1 Baseline characteristics of both groups

\begin{tabular}{|c|c|c|c|}
\hline Characteristics & ECF group $(\mathrm{N}=1,862)$ & Control group $(\mathrm{N}=1,862)$ & $P$ value \\
\hline Body-mass index, $\mathrm{kg} / \mathrm{m}^{2}$ & $22.44 \pm 2.97$ & $22.45 \pm 2.89$ & 0.861 \\
\hline Type of infertility, No. (\%) & & & 1.000 \\
\hline Primary infertility & $979(52.58)$ & $979(52.58)$ & \\
\hline Indications for IVF/ICSI, No. (\%) & & & 1.000 \\
\hline Tubal factor & $545(29.27)$ & $545(29.27)$ & \\
\hline Male factor & $254(13.53)$ & $254(13.53)$ & \\
\hline Combined factors & $1,063(57.09)$ & $1,063(57.09)$ & \\
\hline Baseline follicle-stimulating hormone, IU/mL & $6.77 \pm 1.58$ & $6.76 \pm 1.66$ & 0.990 \\
\hline Baseline luteinizing hormone, IU/L & $5.54 \pm 3.52$ & $5.59 \pm 3.44$ & 0.674 \\
\hline Baseline estradiol, pmol/L & $36.20(27.50-48.40)$ & $36.50(28.30-47.53)$ & 0.638 \\
\hline
\end{tabular}

Plus-minus values are means $\pm \mathrm{SD}$. ECF, endometrial cavity fluid.

pathology and is thought to have no negative effect on the IVF pregnancy rate (13). Our data showed that the toxic effects of hydrosalpinx on embryos and gametes may further aggravate the adverse effects of uterine effusion, which consistent with previous studies (14). There are two different views about the ingredients that may be contained in ECF. Some studies have speculated that the presence of ECF dilutes putatively beneficial factors in the uterus following ET (15). There are also changes in the expression levels of genes associated with uterine receptivity (e.g., integrin $\alpha v \beta 3$ ) (16). However, other studies suggest that growth factors from hydrosalpinx fluid might play a positive role during implantation $(11,17)$.

The purpose of the present study was to investigate the association between the presence of ECF and pregnancy outcomes in patients subjected to IVF/ICSI. All patients with ECF included in this study were identified during $\mathrm{COH}$, and the amount of fluid did not reach the routine treatment standard of our hospital $(\leq 3.5 \mathrm{~mm})$. The clinical pregnancy and live birth rates of patients with ECF were significantly worse than those of the controls.

We also explored the impact of ECF on obstetric outcomes. The main indices of obstetric outcome (including gestational age, birth weight, fetal gender, dysplasia, and number of fetuses) showed no significant differences between the groups. After implantation, the environment in the uterus changes accordingly. The formation of pinopodes promotes the absorption of fluid in the uterine cavity. This may be the mechanism by which uterine effusion does not continue to affect the growing embryo. We also found a higher incidence of gestational diabetes in the ECF group, which is considered to be one of the causes of polyhydramnios (18). The results of previous studies have shown that endometrial fluid contains significantly different protein compositions during the implantation and non-implantation cycles, and that endometrial fluid during the non-implantation cycle increases inflammation and impaired glucose metabolism (19). We speculate that persistent uterine effusion may itself be an indicator of a non-implantable endometrial state. However, the possible causal relationship between short-term changes in blood glucose levels and the accumulation of ECF requires further study. To our knowledge, this is the first report on the relationship between ECF and such obstetric outcomes.

Proper treatment of ECF affects the success of ET and the final pregnancy outcomes of patients subjected to IVF/ ICSI. It has been suggested that ECF should be treated individually according to the causes, appearance, time, and amount in patients undergoing IVF/ICSI; an excess of $3.5 \mathrm{~mm}$ is considered the standard for determining whether 
Table 2 Outcomes of controlled ovarian hyperstimulation $(\mathrm{COH})$

\begin{tabular}{|c|c|c|c|}
\hline Characteristics & ECF group $(\mathrm{N}=1,862)$ & Control group $(\mathrm{N}=1,862)$ & $P$ value \\
\hline IVF & $1,335(71.70)$ & $1,315(70.62)$ & \\
\hline ICSI & $485(26.05)$ & $508(27.28)$ & \\
\hline IVF/ICSI & $42(2.26)$ & $39(2.09)$ & \\
\hline Protocol of $\mathrm{COH}$, No. (\%) & & & 1.000 \\
\hline Short agonist & $128(6.87)$ & $128(6.87)$ & \\
\hline Long agonist & $1,716(92.16)$ & $1,716(92.16)$ & \\
\hline Others & $18(0.97)$ & $18(0.97)$ & \\
\hline Total dosage of $\mathrm{GnRH}$, IU & $2,030.18 \pm 796.52$ & $1,997.07 \pm 779.15$ & 0.200 \\
\hline Estradiol level on hCG trigger day, $\mathrm{pmol} / \mathrm{L}$ & $4,202.73 \pm 2,137.12$ & $4,251.05 \pm 2,103.27$ & 0.487 \\
\hline Progesterone level on hCG trigger day, ng/mL & $0.88(0.68-1.13)$ & $0.90(0.70-1.16)$ & 0.008 \\
\hline Endometrial thickness on $\mathrm{hCG}$ trigger day, $\mathrm{cm}$ & $1.14 \pm 0.15$ & $1.14 \pm 0.16$ & 0.587 \\
\hline No. of oocytes retrieved & $12.45 \pm 5.09$ & $12.44 \pm 5.26$ & 0.955 \\
\hline No. of good-quality embryos & $4.43 \pm 2.54$ & $4.47 \pm 2.55$ & 0.059 \\
\hline No. of embryos transferred & $2.01 \pm 0.40$ & $2.01 \pm 0.38$ & 1.000 \\
\hline \multicolumn{4}{|l|}{ Fertilization rate, $\%$} \\
\hline
\end{tabular}

Plus-minus values are means \pm SD. IVF, in vitro fertilization; ICSI, intracytoplasmic sperm injection; GnRH, gonadotropin-releasing hormone; hCG, human chorionic gonadotropin; ECF, endometrial cavity fluid.

Table 3 Comparison of pregnancy outcomes between the two groups

\begin{tabular}{|c|c|c|c|}
\hline Outcome & ECF group $(\mathrm{N}=1,862)$ & Control group $(\mathrm{N}=1,862)$ & $P$ value \\
\hline Clinical pregnancy rate ${ }^{\ddagger}$ & $56.98 \%(1,061 / 1,862)$ & $63.48 \%(1,182 / 1,862)$ & $<0.001$ \\
\hline Biochemical pregnancy rate ${ }^{\S}$ & $63.48 \%(1,182 / 1,862)$ & $69.17 \%(1,288 / 1,862)$ & $<0.001$ \\
\hline Embryo implantation rate" & $40.11 \%(1,500 / 3,740)$ & $44.41 \%(1,661 / 3,740)$ & $<0.001$ \\
\hline Abortion during biochemical pregnancy & $10.24 \%(121 / 1,182)$ & $8.23 \%(106 / 1,288)$ & 0.085 \\
\hline Abortion during clinical pregnancy & $12.44 \%(132 / 1,061)$ & $10.15 \%(120 / 1,182)$ & 0.087 \\
\hline
\end{tabular}


Table 4 Obstetrical outcomes of patients with live birth in both groups

\begin{tabular}{|c|c|c|c|}
\hline Outcome & ECF group $(\mathrm{N}=902)$ & Control group $(\mathrm{N}=1,033)$ & $P$ value \\
\hline Delivery method & & & 0.788 \\
\hline Transvaginal delivery & $14.86 \%(134 / 902)$ & $15.30 \%(158 / 1,033)$ & \\
\hline Cesarean section & $85.14 \%(768 / 902)$ & $84.70 \%(875 / 1,033)$ & \\
\hline Pregnancy-induced hypertension & $43.59 \%(34 / 78)$ & $55.32 \%(52 / 94)$ & 0.126 \\
\hline Gestational diabetes & $21.79 \%(17 / 78)$ & $8.51 \%(8 / 94)$ & 0.014 \\
\hline Abnormal placenta & $11.54 \%(9 / 78)$ & $9.57 \%(9 / 94)$ & 0.675 \\
\hline Anemia & $20.51 \%(16 / 78)$ & $13.83 \%(13 / 94)$ & 0.244 \\
\hline Male & $52.25 \%(622 / 1,267)$ & $52.27 \%(725 / 1,387)$ & \\
\hline Female & $47.75 \%(605 / 1,267)$ & $47.72 \%(662 / 1,387)$ & \\
\hline Dysplasia & $0.08 \%(1 / 1,267)$ & $0.43 \%(6 / 1,387)$ & 0.076 \\
\hline No. of fetuses & & & 0.862 \\
\hline Single pregnancy & $64.19 \%(579 / 902)$ & $64.57 \%(667 / 1,033)$ & \\
\hline Multiple pregnancy & $35.81 \%(323 / 902)$ & $35.43 \%(366 / 1,033)$ & \\
\hline
\end{tabular}

Plus-minus values are means $\pm \mathrm{SD}$. ECF, endometrial cavity fluid.

ECF should be processed (20). One study concluded that ECF should be removed with a catheter immediately before ET (21). Another study developed a flow chart for how to deal with ECF in different situations; briefly, cryopreservation of the embryos should be considered when there is a large amount of ECF or when this issue is combined with a complex intrauterine environment (22).

Clearly, further research into the treatment of ECF is needed. According to our study, we believe the presence of ECF is an indicator of adverse pregnancy outcomes even when the amount is less than $3.5 \mathrm{~mm}$ in the anteroposterior dimension. Our previous experience shows that in addition to transvaginal sonographic ECF aspiration, moderate curettage of the uterus is also effective. We also found that some Chinese traditional herbal medicines such as Leonurus cardiaca might help with the absorption of effusions in some cases. We recommend processing ECF immediately to avoid postponing ET, which places economic and psychological pressures on patients.

There were some limitations in our research. Firstly, during the follow-up, some patients could not be contacted for various reasons or could not provide accurate and useful information after being contacted, including some patients diagnosed with ECF in the early phase of data collection. This missing information might have affected the statistical evaluation of the results. Also, in terms of the amount of uterine effusion, there was only one approximate value in the patient information recorded by our hospital, and there was no accurate numerical value to analyze the relationship between the volume of ECF and pregnancy outcomes in a systematic manner. These limitations need to be improved in future research.

Our findings indicate that the presence of ECF is associated with adverse pregnancy outcomes following IVF/ICSI and ET. The clinical significance of this issue is far-reaching, and should arouse the attention of clinicians and be studied extensively. The mechanism of ECF accumulation, as well as the relationship between its amount, timing, and pregnancy outcomes requires further in-depth studies. 


\section{Conclusions}

ECF detected by ultrasonography during $\mathrm{COH}$ had a negative association with IVF/ICSI pregnancy outcomes but did not have a significant association with adverse obstetric outcomes (except for gestational diabetes). Thus, the presence of ECF might be an indicator for the occurrence adverse pregnancy outcomes. The decision to treat uterine effusions should accord with individual patient conditions. The mechanism of ECF production and treatment requires further research.

\section{Acknowledgments}

The authors thank Elsevier Language Editing Services for help in revising the manuscript. We are very grateful to our patients and all participants for their involvement in the process of data collection. We thank Dr. Tang Rong for his help with in vitro fertilization (IVF)/intracytoplasmic sperm injection (ICSI)-related data.

Funding: This study was supported by the National Key Research and Development Program of China (No. 2018YFC1004800) and the National Natural Science Foundation of China (No. 81571414).

\section{Footnote}

Reporting Checklist: The authors have completed the STROBE reporting checklist. Available at http://dx.doi. org/10.21037/atm-20-3623

Data Sharing Statement: Available at http://dx.doi. org/10.21037/atm-20-3623

Peer Review File: Available at http://dx.doi.org/10.21037/ atm-20-3623

Conflicts of Interest: All authors have completed the ICMJE uniform disclosure form (available at http://dx.doi. org/10.21037/atm-20-3623). The authors have no conflicts of interest to declare.

Ethical Statement: The authors are accountable for all aspects of the work in ensuring that questions related to the accuracy or integrity of any part of the work are appropriately investigated and resolved. The study was approved by the Institutional Review Board of the Reproductive Hospital Affiliated to Shandong University
\{[2018] IRB No. [47]\}. All procedures performed in this study involving human participants were in accordance with the Declaration of Helsinki (as revised in 2013). Due to the retrospective nature of the study informed consent was not required.

Open Access Statement: This is an Open Access article distributed in accordance with the Creative Commons Attribution-NonCommercial-NoDerivs 4.0 International License (CC BY-NC-ND 4.0), which permits the noncommercial replication and distribution of the article with the strict proviso that no changes or edits are made and the original work is properly cited (including links to both the formal publication through the relevant DOI and the license). See: https://creativecommons.org/licenses/by-nc-nd/4.0/.

\section{References}

1. Kuijsters NPM, Methorst WG, Kortenhorst MSQ, et al. Uterine peristalsis and fertility: current knowledge and future perspectives: a review and meta-analysis. Reprod Biomed Online 2017;35:50-71.

2. Salamonsen LA, Evans J, Nguyen HP, et al. The Microenvironment of Human Implantation: Determinant of Reproductive Success. Am J Reprod Immunol 2016;75:218-25.

3. Liu S, Shi L, Shi J. Impact of endometrial cavity fluid on assisted reproductive technology outcomes. Int J Gynaecol Obstet 2016;132:278-83.

4. Chien LW, Au HK, Xiao J, et al. Fluid accumulation within the uterine cavity reduces pregnancy rates in women undergoing IVF. Hum Reprod 2002;17:351-6.

5. He RH, Gao HJ, Li YQ, et al. The associated factors to endometrial cavity fluid and the relevant impact on the IVF-ET outcome. Reprod Biol Endocrinol 2010;8:46.

6. Lee RK, Yu SL, Chih YF, et al. Effect of endometrial cavity fluid on clinical pregnancy rate in tubal embryo transfer (TET). J Assist Reprod Genet 2006;23:229-34.

7. Chen ZJ, Shi Y, Sun Y, et al. Fresh versus Frozen Embryos for Infertility in the Polycystic Ovary Syndrome. N Engl J Med 2016;375:523-33.

8. Gupta N, Bhandari S, Agrawal P, et al. Effect of Endometrial Cavity Fluid On Pregnancy Rate of Fresh Versus Frozen In Vitro Fertilization Cycle. J Hum Reprod Sci 2017;10:288-92.

9. Levi AJ, Segars JH, Miller BT, et al. Endometrial cavity fluid is associated with poor ovarian response and increased cancellation rates in ART cycles. Hum Reprod 
2001;16:2610-5.

10. Bloechle M, Schreiner T, Lisse K. Recurrence of hydrosalpinges after transvaginal aspiration of tubal fluid in an IVF cycle with development of a serometra. Hum Reprod 1997;12:703-5.

11. Granot I, Dekel N, Segal I, et al. Is hydrosalpinx fluid cytotoxic? Hum Reprod 1998;13:1620-4.

12. Strandell A, Sjogren A, Bentin-Ley U, et al. Hydrosalpinx fluid does not adversely affect the normal development of human embryos and implantation in vitro. Hum Reprod 1998;13:2921-5.

13. Polat M, Boynukalin FK, Yarali I, et al. Transient intrauterine fluid accumulation not due to hydrosalpinx or any identifiable pelvic pathology is not detrimental to IVF outcome. Arch Gynecol Obstet 2014;290:569-73.

14. Fleming C, Hull MG. Impaired implantation after in vitro fertilisation treatment associated with hydrosalpinx. Br J Obstet Gynaecol 1996;103:268-72.

15. Lu S, Peng H, Zhang H, et al. Excessive intrauterine fluid cause aberrant implantation and pregnancy outcome in mice. PLoS One 2013;8:e78446.

16. Lessey BA, Castelbaum AJ, Buck CA, et al. Further characterization of endometrial integrins during the menstrual cycle and in pregnancy. Fertil Steril

Cite this article as: Zhang WX, Cao LB, Zhao Y, Li J, Li BF, Lv JN, Yan L, Ma JL. Endometrial cavity fluid is associated with deleterious pregnancy outcomes in patients undergoing in vitro fertilization/intracytoplasmic sperm injection: a retrospective cohort study. Ann Transl Med 2021;9(1):9. doi: 10.21037/atm20-3623
1994;62:497-506.

17. Sawin SW, Loret de Mola JR, Monzon-Bordonaba F, et al. Hydrosalpinx fluid enhances human trophoblast viability and function in vitro: implications for embryonic implantation in assisted reproduction. Fertil Steril 1997;68:65-71.

18. Hamza A, Herr D, Solomayer EF, et al. Polyhydramnios: Causes, Diagnosis and Therapy. Geburtshilfe Frauenheilkd 2013;73:1241-6.

19. Azkargorta M, Escobes I, Iloro I, et al. Differential proteomic analysis of endometrial fluid suggests increased inflammation and impaired glucose metabolism in nonimplantative IVF cycles and pinpoints PYGB as a putative implantation marker. Hum Reprod 2018;33:1898-906.

20. He RH, Zhu XM. How to deal with fluid in the endometrial cavity during assisted reproductive techniques. Curr Opin Obstet Gynecol 2011;23:190-4.

21. Griffiths AN, Watermeyer SR, Klentzeris LD. Fluid within the endometrial cavity in an IVF cycle--a novel approach to its management. J Assist Reprod Genet 2002;19:298-301.

22. Mittal M, Becker CM. How to Manage the Patient with Fluid in the Endometrium Prior to Embryo Transfer 2018:79-85. 